\title{
A Comparative Study on Asperity Peak Modeling Methods
}

\author{
Wei Zhou ${ }^{1 *}$ (i), Daiyan Zhao ${ }^{1}$, Jinyuan Tang ${ }^{2}$ and Jun Yi ${ }^{1}$
}

\begin{abstract}
The peak identification scheme based method (three-point definition) and the spectral moments based method (spectral moment approach) are both widely used for asperity peak modeling in tribology. To discover the differences between the two methods, a great number of rough surface profile samples with various statistical distributions are first randomly generated using FFT. Then the distribution parameters of asperity peaks are calculated for the generated samples with both methods. The obtained results are compared and verified by experiment. The variation rules of the differences between the two methods with statistical characteristics of rough surfaces are investigated. To explain for the discovered differences, the assumptions by spectral moment approach that the joint distribution of surface height, slope and curvature is normal and that the height distribution of asperities is Gaussian, are examined. The results show that it is unreasonable to assume a joint normal distribution without inspecting the correlation pattern of $[z],\left[z^{\prime}\right]$ and $\left[z^{\prime \prime}\right]$, and that the height distribution of asperities is not exactly Gaussian before correlation length of rough surface increases to a certain extent, 20 for instance.
\end{abstract}

Keywords: Surface topography, Roughness, Surface analysis, Asperity peak, Random process model

\section{Introduction}

Surface micro-topography has great influence on contact performances, e.g., friction and wear [1], contact fatigue [2], heat and electric conduction [3]. To evaluate such effects, the well known GW model [4] and its modified versions $[5,6]$ have been developed based on Hertz contact mechanics, where rough surface contact is regarded as asperity contacts. Therefore, the modeling of asperity is of great importance.

Although surface micro-topographies from various machining methods [7] are somewhat different, they are usually characterized and modeled by random process theory due to their statistical characteristics. Longuet-Higgins [8] and Nayak [9] treated surface microtopography as a random process, and used height distribution and auto-correlation function (ACF) to analyze

\footnotetext{
*Correspondence: 1030140@hnust.edu.cn

1 Hunan Provincial Key Laboratory of High Efficiency and Precision Machining of Difficult-to-Cut Material, Hunan University of Science and Technology, Xiangtan 411201, China

Full list of author information is available at the end of the article
}

the sequences of sampled points. For the convenience of research and based on practical cognition, Gaussian height distribution and exponential ACF are commonly used to characterize rough surfaces, with which Nayak [9] and McCool [10] modeled asperity distribution by spectral moment approach. In this way, the mathematic formulae of distribution parameters were deduced for asperity peak and asperity summit. Recently, the uncertainty in the calculation of asperity distribution parameters by spectral moment approach was discovered by Pawar et al. [11]. It was noted that the calculated asperity distribution parameters of samples with the same height distribution and ACF could vary within a certain range, and that such a variation decreased with increasing correlation length. Subsequently, the research by Zhou et al. [12] indicated that the assumption that the joint distribution of surface height, slope and curvature was normal could bring modeling deviation to spectral moment approach in comparison with another commonly used method, namely, the asperity identification scheme based method, i.e., three-point definition. The effect of asperity 
peak definition on its properties with different numbers of neighboring points was reported by Pogačnik and Kalin [13]. Kalin et al. [14], however, believed the fact that not only asperity peaks but also asperity shoulders were considered accounted for the modeling error of spectral moment approach. In addition, the characterization of rough surfaces using spectral moment was reported by Panda et al. [15].

Besides the assumption on the joint distribution of surface height, slope and curvature, the distribution of asperity heights is assumed to be Gaussian. With such an assumption, the mean of asperity heights, the root mean square (RMS) of asperity height distribution and the average curvature radius of asperities were derived using spectral moments [16]. The discussion about the effect of bi-Gaussian rough surface on asperity contact was carried out by $\mathrm{Hu}$ et al. [17]. In case of surface with fractal characteristic, fractal theory is utilized to develop the asperity model [18].

In consideration of the extensive application of both three-point definition and spectral moment approach, to examine their validity and to discover their differences are beneficial to effective modeling for asperity peak. To this end, a great number of rough surface profile samples with various statistical distributions are first randomly generated using FFT. Then the distribution parameters of asperity peaks are calculated for the generated samples with both methods. The obtained results are compared and verified by experiment. The effects of standard length, and $\beta$ is correlation length. It should be noted that $[z]$ is actually Gaussian white noise when $\beta=0 . \vartheta$ is high pass filtering constant, representing high pass filtering with different cut-off lengths. Obviously, correlation decays faster with a larger absolute value of $\vartheta$. Whitehouse and Archard [19] defined correlation length as the distance where $R$ decayed to $1 / 10$ of the origin value. This corresponds to $\vartheta=-2.3$. Besides, $\vartheta=-0.844$ and $\vartheta=-1$ were used by Hirst and Hollander [20] and Aramaki et al. [21] respectively. These definitions will all be chosen for investigating the effect of $\vartheta$.

In order for the generation of $[z]$ with given statistical distributions, a pseudorandom number generator is used to generate Gaussian white noise sequence $[\varepsilon]$ with a specified standard deviation $\sigma$. To go further, a convolution operation is performed on the white noise sequence and on the filter coefficient $[h]$ decided by ACF. That is

$$
z(i)=\sum_{k=-\frac{T}{2}+1}^{\frac{T}{2}-1} h(k) \varepsilon(i+k)
$$

where $T$ is ACF truncation length and the filter coefficient $h(k)$ is

$$
h(k)=\frac{1}{T} \sum_{\omega=-\frac{T}{2}+1}^{\frac{T}{2}-1} H(\omega) \exp (-j k \omega) .
$$

To determine the frequency response $H$, the Fourier transform of ACF is first calculated:

$$
S(\omega)=\frac{1}{T} \sum_{k=-\frac{T}{2}+1}^{\frac{T}{2}-1} R(k) \exp (-j k \omega), \omega=-T / 2+1, \cdots, T / 2-1,
$$

deviation, correlation length and high pass filtering constant of rough surface on the differences between the two methods are investigated. Finally, discussions are made to explain for the discovered differences.

\section{Methodology}

2.1 Generation of Rough Surface Profile Samples

Rough surfaces with Gaussian height distribution and exponential ACF are expressed as

$$
\left\{\begin{array}{l}
\phi(z)=\frac{1}{\sqrt{2 \pi} \sigma} \exp \left(-\frac{(z-\mu)^{2}}{2 \sigma^{2}}\right), \\
R(\tau)=\sigma^{2} \exp [\vartheta \cdot(\tau / \beta)],
\end{array}\right.
$$

where $z$ is surface height; $\mu, \sigma$ and $\phi$ are the mean, the standard deviation and the probability density function (PDF) of $[z]$, respectively; $R$ is auto-correlation, $\tau$ is lag where $S(\omega)$ is the power spectral density.

According to the definition of ACF and Eq. (2), it gives

$$
H(\omega)=\sqrt{S(\omega) / C}
$$

where $C$ is a constant, the Fourier transform of the sequence $[\varepsilon]$. Thereupon, any desired height sequence $[z]$ with prescribed $\sigma$ and $\beta$ can be generated.

The detailed theory and methodology for rough surface simulation based on FFT can be found in Ref. [22]. It was suggested by $\mathrm{He}$ et al. [23] that the ratio of ACF truncation length $T$ to correlation length $\beta$ should be greater than 6 so that the asperity distribution parameters could converge to their true values. 


\subsection{Modeling of Asperity Peak Distribution}

According to three-point definition of asperity peak, any point $z_{i}$ from $[z]$ becomes a peak when

$$
z_{i}>z_{i-1} \& z_{i}>z_{i+1} \text {. }
$$

All the heights of $z$ satisfying Eq. (6) form the height distribution of asperity peaks $[\xi]$. The mean $\xi_{\text {avg }}$, the root mean square (RMS) $\sigma_{\xi}$, the skewness $\xi_{\text {sk }}$ and the kurtosis $\xi_{\mathrm{ku}}$ of asperity height distribution can be calculated according to their definitions, i.e.,

$$
\left\{\begin{array}{l}
\xi_{\text {avg }}=\frac{1}{n_{\mathrm{p}}} \sum_{i=1}^{n_{\mathrm{p}}} \xi_{i}, \\
\sigma_{\xi}=\sqrt{\frac{1}{n_{\mathrm{p}}} \sum_{i=1}^{n_{\mathrm{p}}}\left(\xi_{i}-\xi_{\mathrm{avg}}\right)^{2},} \\
\xi_{\mathrm{sk}}=\frac{1}{n_{\mathrm{p}} \sigma_{\xi}^{3}} \sum_{i=1}^{n_{\mathrm{p}}}\left(\xi_{i}-\xi_{\mathrm{avg}}\right)^{3}, \\
\xi_{\mathrm{ku}}=\frac{1}{n_{\mathrm{p}} \sigma_{\xi}^{4}} \sum_{i=1}^{n_{\mathrm{p}}}\left(\xi_{i}-\xi_{\mathrm{avg}}\right)^{4},
\end{array}\right.
$$

where $n_{\mathrm{p}}$ is the number of asperity peaks.

The curvature of asperity peak is

$$
\kappa=\left(-z_{i+1}+2 z_{i}-z_{i-1}\right) / d^{2},
$$

where $d$ is sampling interval. The curvature radius of asperity peak is

$$
r_{\mathrm{p}}=1 / \kappa,
$$

The density of asperity peak is

$$
\eta=\frac{n_{\mathrm{p}}}{L}=\frac{n_{\mathrm{p}}}{N \cdot d}
$$

where $L$ is sampling length and $N$ is the number of sampling points. Unit sampling interval will be used to calculate $r_{\mathrm{p}}$ and $\eta$ in the following, and non-dimensional results will be obtained.

In addition to the peak identification scheme based method, a mathematical model of asperity peak distribution was established by Nayak [9] based on central moments of surface power spectral density (PSD) using random process theory, namely spectral moment approach. The assumption that the joint distribution of surface height, slope and curvature was normal was made during the derivation. From the deduced model, the height distribution of asperity peaks is

$$
\begin{aligned}
& \phi_{\text {peak }}\left(\xi^{*}\right)=\frac{\delta}{\sqrt{2} \pi}\left\{\exp \left[-\left(\xi^{* 2} / 2 \delta^{2}\right)\right]+\right. \\
& \left.\sqrt{\pi} \chi \exp \left(-0.5 \xi^{* 2}\right)(1+\operatorname{erf} \chi)\right\},
\end{aligned}
$$

where $\xi^{*}=\xi / \sigma, \alpha$ is band width coefficient and $\alpha=m_{0} m_{4} / m_{2}^{2}, \quad \delta=[(\alpha-1) / \alpha]^{1 / 2}, \quad \chi=\left(\frac{1-\delta}{2 \delta^{2}}\right)^{1 / 2} \xi^{*}$, and the central moments of order zero to order four of PSD are

$$
\begin{aligned}
& m_{0}=R(0)=\sigma^{2}, \\
& m_{2}=\frac{\mathrm{d}^{2} R(0)}{\mathrm{d} \tau^{2}}, \\
& m_{4}=\frac{\mathrm{d}^{4} R(0)}{\mathrm{d} \tau^{4}},
\end{aligned}
$$

where $m_{2}$ and $m_{4}$ are RMS slope and RMS curvature of rough surface profile, respectively.

The curvature distribution of asperity peaks is

$$
\bar{\kappa}\left(\xi^{*}\right)=\sqrt{2} m_{4}^{1 / 2} \delta \frac{\left[\chi+\sqrt{\pi} \exp \left(\chi^{2}\right)(1+\operatorname{erf} \chi)\left(\chi^{2}+0.5\right)\right]}{\left[1+\chi \exp \left(\chi^{2}\right) \sqrt{\pi}(1+\operatorname{erf} \chi)\right]} .
$$

Substituting the mean height of asperity peaks into Eq. (13), the average curvature radius of asperity peaks can be calculated approximately as

$$
r_{\mathrm{p}}=\frac{1}{\bar{\kappa}\left(\xi_{\mathrm{avg}} / \sigma\right)} .
$$

The density of asperity peak is

$$
\eta=\frac{1}{2 \pi}\left(m_{4} / m_{2}\right)^{1 / 2} .
$$

On the basis of the work by Nayak, and on the assumption that the height distribution of asperities was Gaussian, the mean and the RMS of asperity height distribution were further obtained by McCool [10] as follows:

$$
\begin{aligned}
& \xi_{\text {avg }}=\frac{4 \sigma}{\sqrt{\pi \alpha}}, \\
& \sigma_{\xi}=(1-0.8968 / \alpha)^{1 / 2} \sigma .
\end{aligned}
$$

\section{Methodology}

\subsection{Simulation Analysis}

To find out the differences between three-point definition and spectral moment approach, rough surface profile samples for the calculation of distribution parameters of asperity peaks are first randomly generated using FFT for comparison. In order to reduce the influence of random error, 100 samples are generated for the same $(\sigma, \beta)$. The mean values of all the samples are taken as the final results. The influences of sampling length and sampling interval are not considered in this paper [24]. The ACFs 
of generated samples and those of theory are plotted in Figure 1 . The simulation shows good accordance with theory either under $\beta=0$ or under $\beta=100$. Recalling that $[z]$ is Gaussian white noise when $\beta=0$, the corresponding simulation error of $[z]$ is totally determined by the generation precision of Gaussian white noise by computer.
Consequently, the program used for the generation of rough surface profile samples in this paper is proved to be reliable.

The PDFs of the height distribution of asperity peaks calculated from three-point definition and spectral moment approach are shown in Figure 2. It is evident
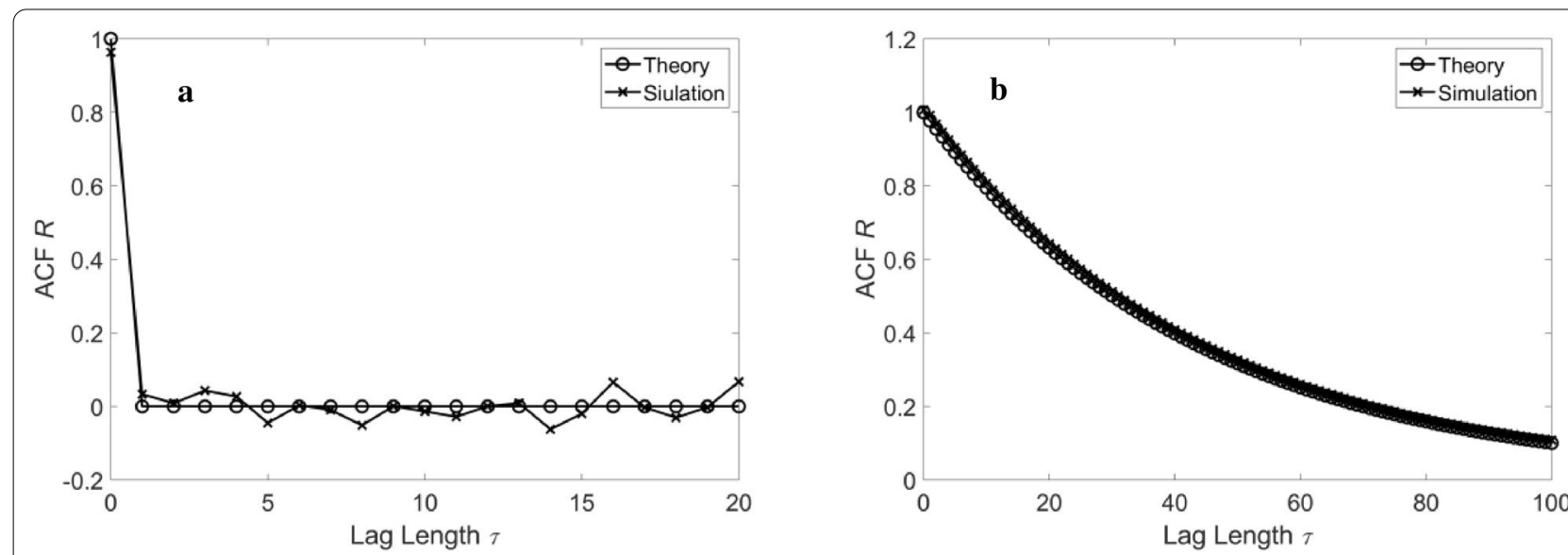

Figure 1 Comparison on ACF between simulation and theory $(\sigma=1, \vartheta=-2.3)$ : (a) $\beta=0,(\mathbf{b}) \beta=100$
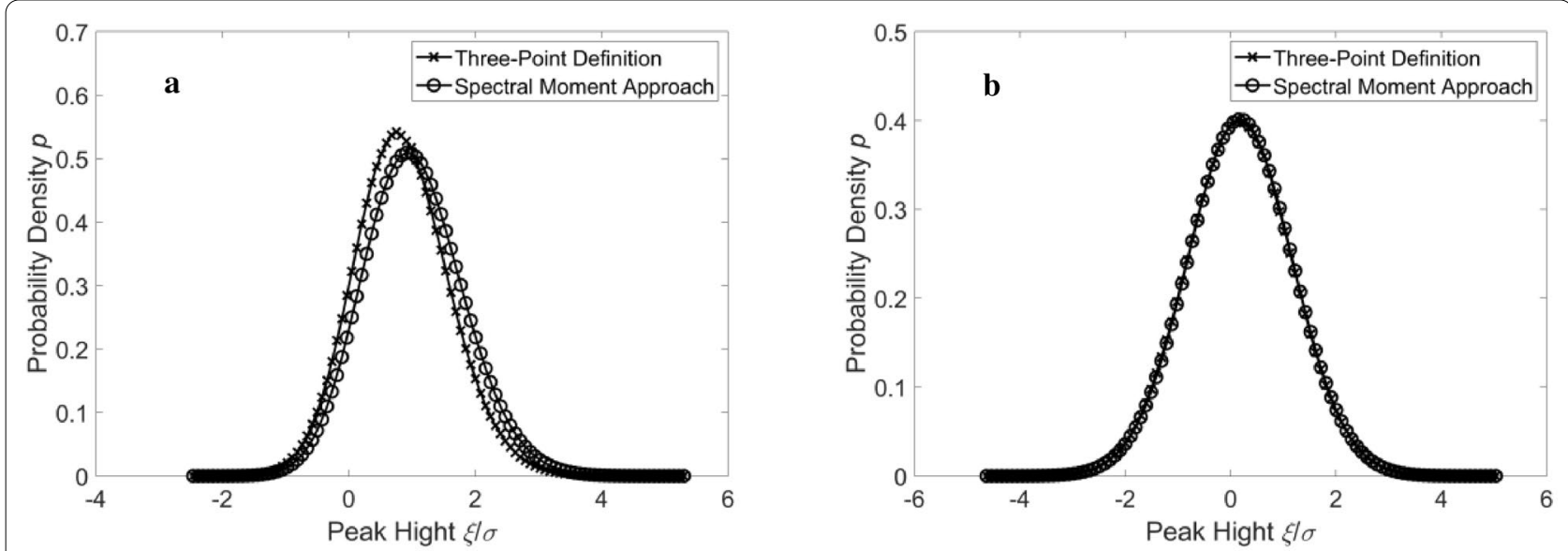

Figure 2 Comparison on PDF of [ $\xi$ ] under different correlation lengths $(\sigma=1, \vartheta=-2.3):(\mathbf{a}) \beta=0$, (b) $\beta=100$

Table 1 Comparison of [द] for $\sigma=1, \vartheta=-2.3$

\begin{tabular}{llllll}
\hline Correlation length & Method & $\boldsymbol{\xi}_{\text {avg }}$ & Percent variation & $\boldsymbol{\sigma}_{\boldsymbol{\xi}}$ & Percent variation \\
\hline 0 & Three-point definition & 0.8445 & 0 & 1.0005 & 0 \\
\multirow{2}{*}{50} & Spectral moment approach & 1.8411 & $118.01 \% \uparrow$ & 0.6352 & $-36.51 \% \downarrow$ \\
& Three-point definition & 0.2366 & 0 & 0.9980 & 0 \\
\multirow{2}{*}{100} & Spectral moment approach & 0.4619 & $95.22 \% \uparrow$ & 0.9833 & $-1.47 \% \downarrow$ \\
& Three-point definition & 0.1890 & 0 & 1.0035 & 0 \\
& Spectral moment approach & 0.2274 & $20.32 \% \uparrow$ & 0.9909 & $-1.26 \% \downarrow$ \\
\hline
\end{tabular}




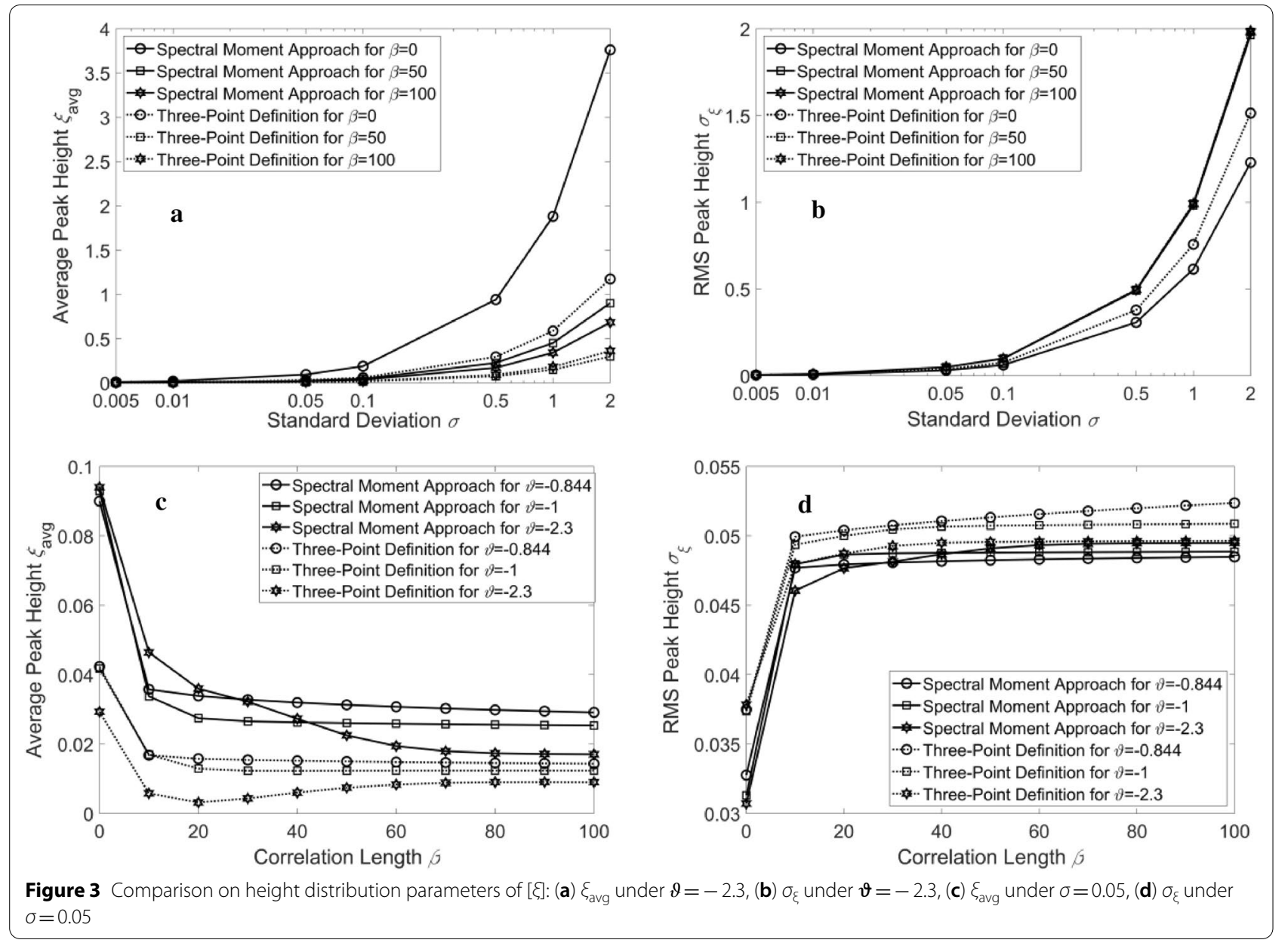

that, compared with three-point definition, spectral moment approach overestimates the mean $\xi_{\text {avg }}$ and underestimates the RMS $\sigma_{\xi}$ of asperity peak height distribution $[\xi]$ at small correlation lengths $(\beta=0)$. As correlation length increases to a certain extent $(\beta=100)$, the PDFs obtained from the two methods almost overlap with each other. Table 1 lists the quantitative differences between the two methods.

The variations of the differences in the calculation of $\xi_{\text {avg }}$ and $\sigma_{\xi}$ between the two methods are shown in Figure 3 , where the abscissa is scaled logarithmically. It is found that standard deviation of rough surface $\sigma$ has great influence on the differences in the calculation of $\xi_{\text {avg }}$ and $\sigma_{\xi}$ by different methods. As $\sigma$ increases, the calculation differences grow. The effect of high pass filtering constant $\vartheta$ depends on correlation length $\beta$. While the calculation differences of $\xi_{\text {avg }}$ and $\sigma_{\xi}$ are greater for smaller $\vartheta$ at small $\beta$, the situation is gradually reversed as $\beta$ increases.
Figure 4 shows the calculated curvature distributions of asperity peaks from the two methods. As can be seen, nearly all the curvatures of asperity peaks are overestimated by spectral moment approach, except for $|\xi / \sigma|>3$ which should be brought about by randomness. Moreover, the calculation difference of asperity peak curvature $\kappa$ increases with the height of asperity peak $\xi$ and decreases with increasing $\beta$. The curvature radius of asperity peak $r_{\mathrm{p}}$, the inverse of $\kappa$, is to be discussed instead in the following for its application in rough surface contact analysis. The variation of the difference in the calculation of the average curvature radius of asperity peaks between the two methods is shown in Figure 5. It can be found that the average curvature radii of asperity peaks calculated from spectral moment approach are smaller than those of three-point definition. The calculation difference of $r_{\mathrm{p}}$ increases with $\beta$ and decreases with increasing $\sigma$. As $\vartheta$ becomes smaller, the calculation difference of $r_{\mathrm{p}}$ gets greater. 

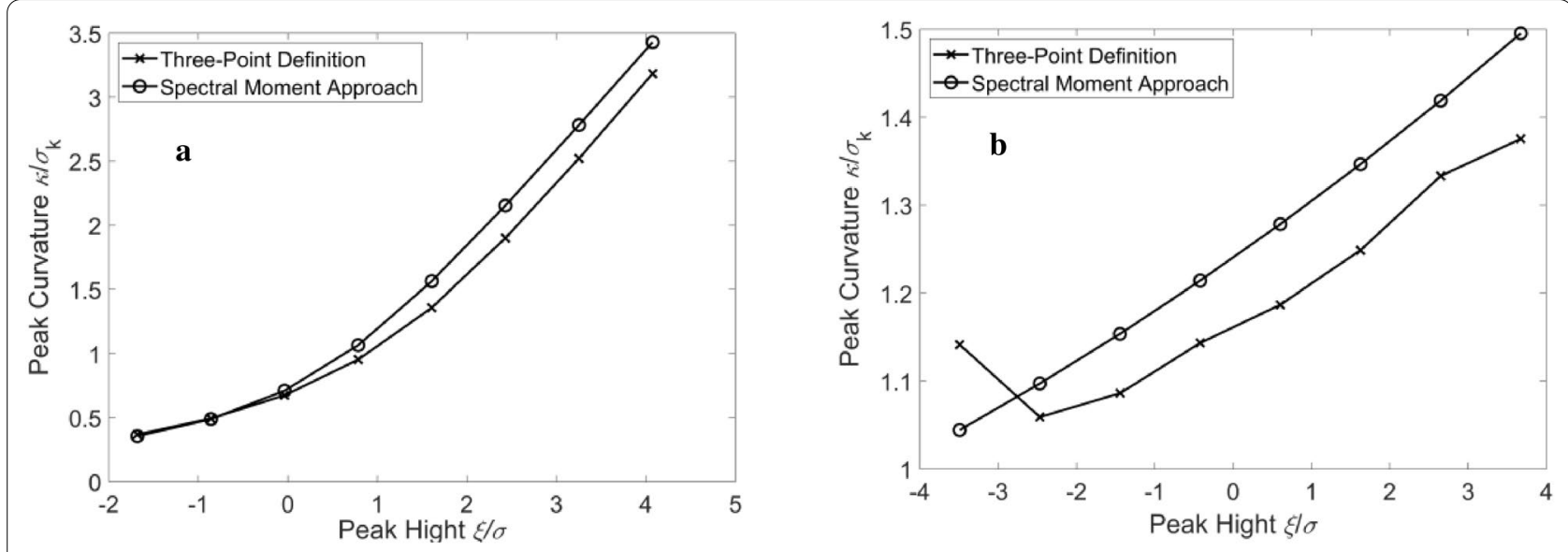

Figure 4 Comparison on curvature distribution of asperity peaks under different correlation lengths $(\sigma=1, \vartheta=-2.3)$ : (a) $\beta=0$, (b) $\beta=100$
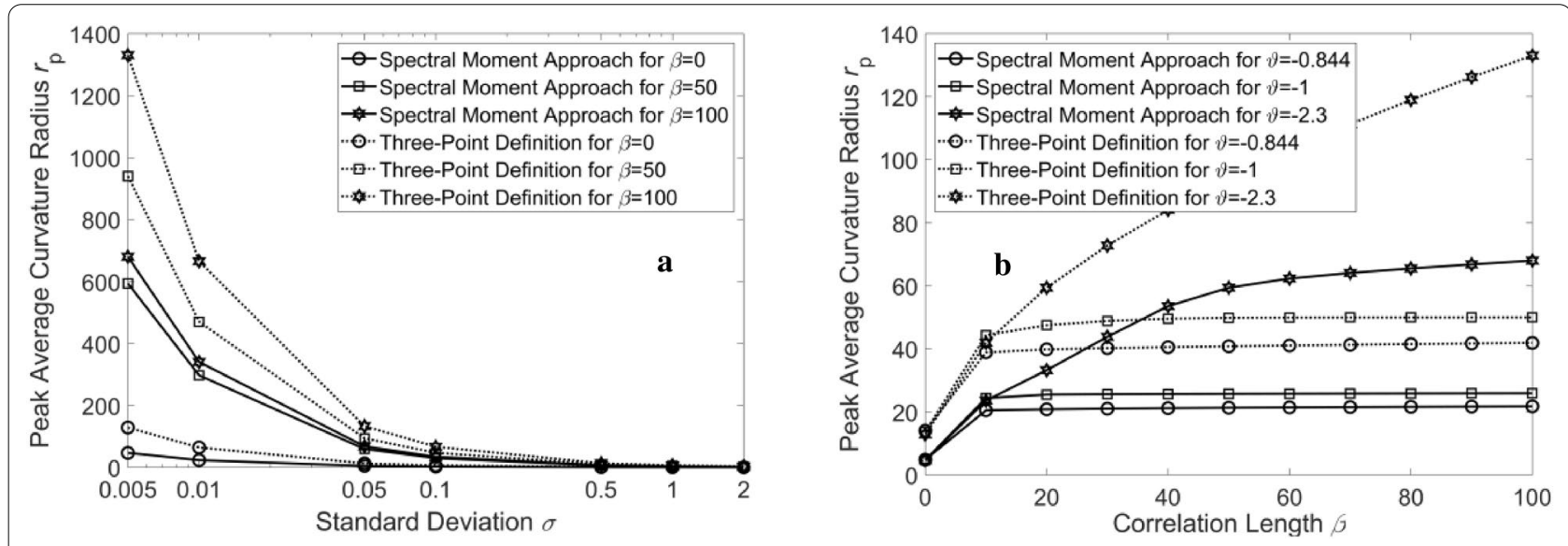

Figure 5 Comparison on average curvature radius of asperity peaks: (a) under $\boldsymbol{\vartheta}=-2.3$, (b) under $\sigma=0.05$

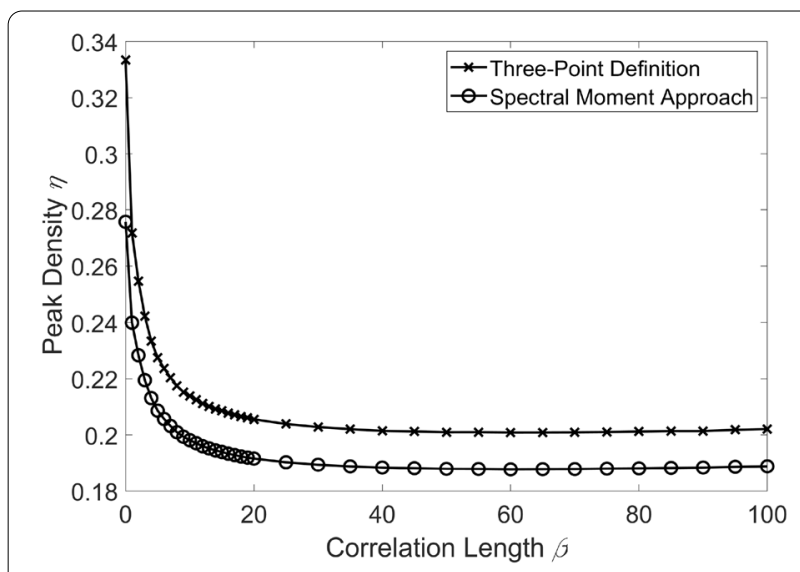

Figure 6 Comparison on asperity peak density $(\sigma=1, \vartheta=-2.3)$
The comparison on asperity peak density $\eta$ is shown in Figure 6 and Figure 7. It can be seen from the figure that, although the calculated $\eta$ from spectral moment approach shows the same change pattern with that of three-point definition, the former is underestimated. With $\beta$ growing, both the calculated $\eta$ values go down and converge to a constant. The calculation difference of $\eta$ tends to increase with $\beta$, and it has nothing to do with $\sigma$. The effect of $\vartheta$ on the calculation of $\eta$ is remarkable. The results state that under $\vartheta=-0.844$ and $\vartheta=-1, \eta$ still decreases with increasing $\beta$ when spectral moment approach is employed, whereas $\eta$ shows an increasing tendency with $\beta$ in the case of using threepoint definition. 

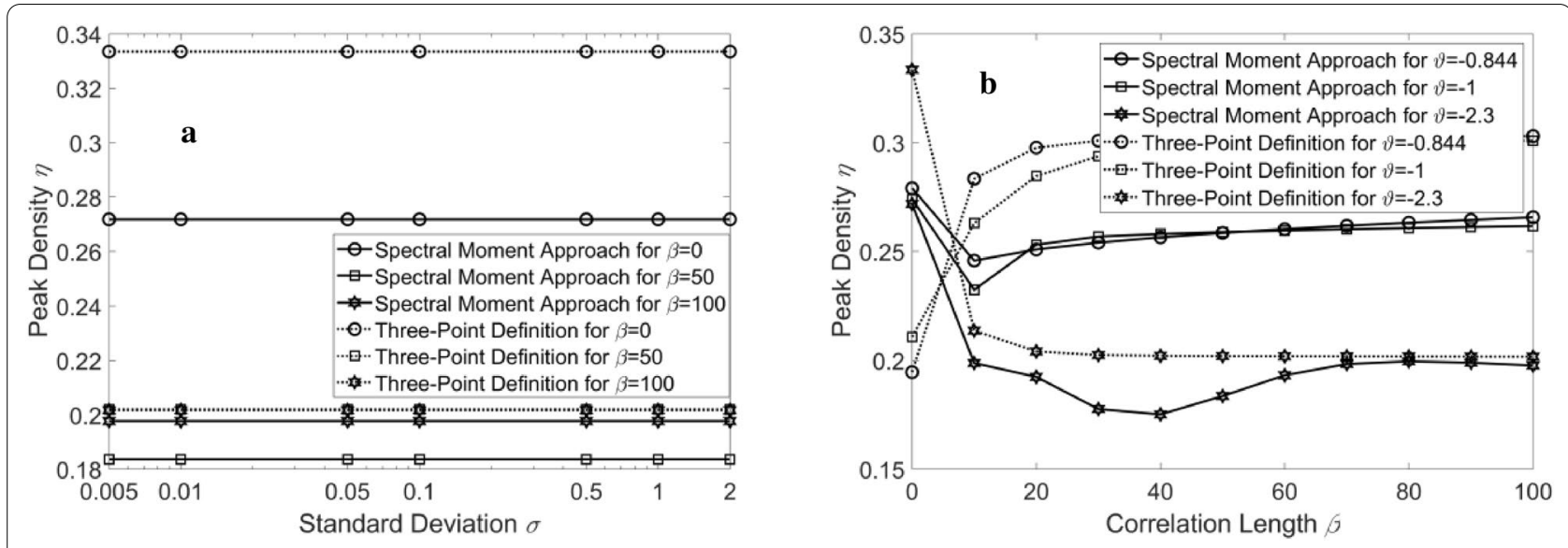

Figure 7 Comparison on asperity peak density: (a) under $\vartheta=-2.3$, (b) under $\sigma=0.05$

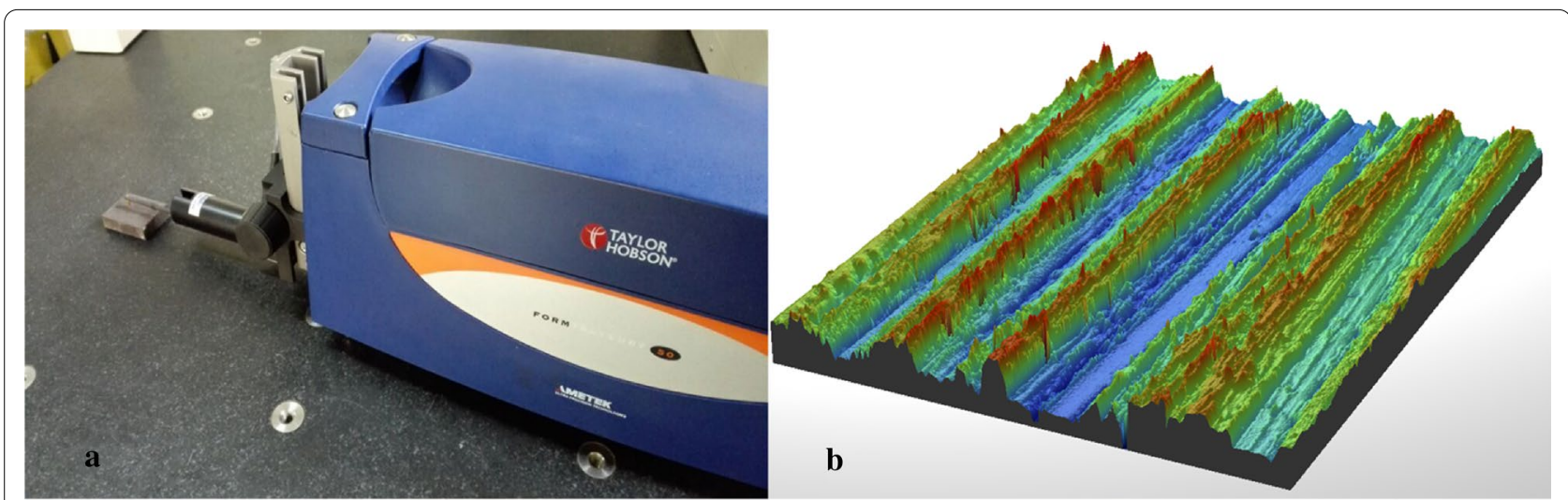

Figure 8 Roughness measuring instrument and tested sample (obtained by scanning with white-light interferometer Veeco Wyko NT9100): (a) Taylor Hobson roughness measuring instrument, (b) surface topography of Specimen \#3

\subsection{Experiment Verification}

To confirm the discovered differences between threepoint definition and spectral moment approach, the rough surfaces of four grinding samples (Specimen \#1-Specimen \#4) are tested by Taylor Hobson roughness measuring instrument, as shown in Figure 8. The samples, made of $45 \#$ steel, are prepared using a surface grinding machine M7130 with an alumina grinding wheel WA46L. The circular linear speed of the grinding wheel is $26 \mathrm{~m} / \mathrm{s}$ while the feed speed of the worktable is $0.069 \mathrm{~m} / \mathrm{s}$. The grinding depths for Specimen \#1-Specimen \#4 are $5 \mathrm{um}, 10 \mathrm{um}, 15 \mathrm{um}$ and $20 \mathrm{um}$, respectively. The test direction is perpendicular to texture direction. To facilitate comparison with simulation analysis, the measured height sequences $[z]$ are normalized with a mean value of 0 and $\sigma=1$.

Note that for a specified correlation distance in physical, correlation length $\beta$ is non-dimensionalized with sampling interval $d$. The larger the sampling interval is, the smaller the correlation length is. To obtain more samples of diverse correlation lengths, the measured height sequences $[z]$ of Specimen \#1-Specimen \#4 are sampled repeatedly by adjusting sampling interval, i.e., sampling $[z]$ every few points to produce new sequences $\left[z^{\prime}\right]$. In this way, a series of tested samples of different correlation lengths are obtained approximately. The sampled topographies of different correlation lengths from Specimen \#3 are shown in Figure 9. The height distribution PDFs and ACFs of rough surfaces from Specimen \#3 and those of theory under different correlation lengths are shown in Figure 10. It can be seen that the hypotheses of Gaussian height distribution and exponential ACF are basically achieved.

On the basis of three-point definition, the height distribution PDFs and curvature distributions of asperity peaks are calculated with the height sequences $[z]$ sampled from Specimen \#3. Meanwhile, the central moments of PSD determined with the same $[z]$ are used to calculate 

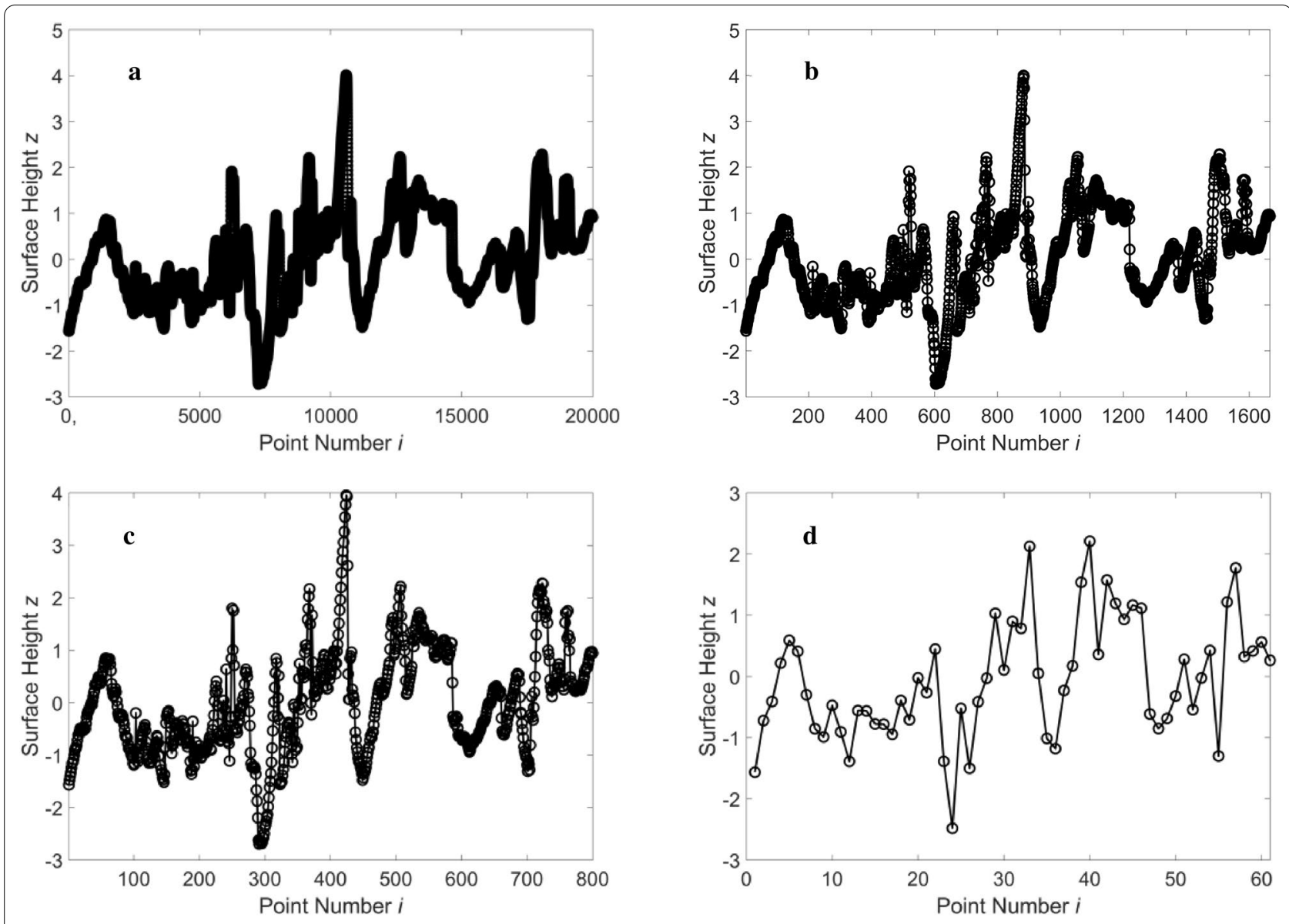

Figure 9 Sampled topographies from Specimen $\# 3(\vartheta=-2.3)$ : (a) $\beta=1298$, (b) $\beta=100,(\mathbf{c}) \beta=50$, (d) $\beta=4$

asperity peak distribution parameters based on spectral moment approach. The results obtained from the two methods are shown in Figure 11 and Figure 12. Table 2 clearly shows that the average height and the curvatures of asperity peaks predicted by spectral moment approach are generally larger than those from experiment. In a few cases, the calculated curvatures of asperity peaks from experiment are larger in comparison with those of spectral moment approach. This is probably due to the fact that the tested samples do not satisfy the assumed ideal statistical distributions exactly. The comparison also confirms the discovery that the RMS of asperity peak height distribution $[\xi]$ is underestimated by spectral moment approach.

\section{Discussions}

Since three-point definition is the most reliable method $[13,25]$ and is more trustworthy than spectral moment approach [11], the deviation of the latter from the former is believed to be the modeling error of asperity peak for spectral moment approach. To account for the differences between the two methods, two assumptions based on which spectral moment approach was developed are to be examined next.

To start with, $[z],\left[z^{\prime}\right]$ and $\left[z^{\prime \prime}\right]$ were all expected to be normal distributions. Without inspecting their correlation pattern, their joint probability density was arbitrarily taken as [9]: 

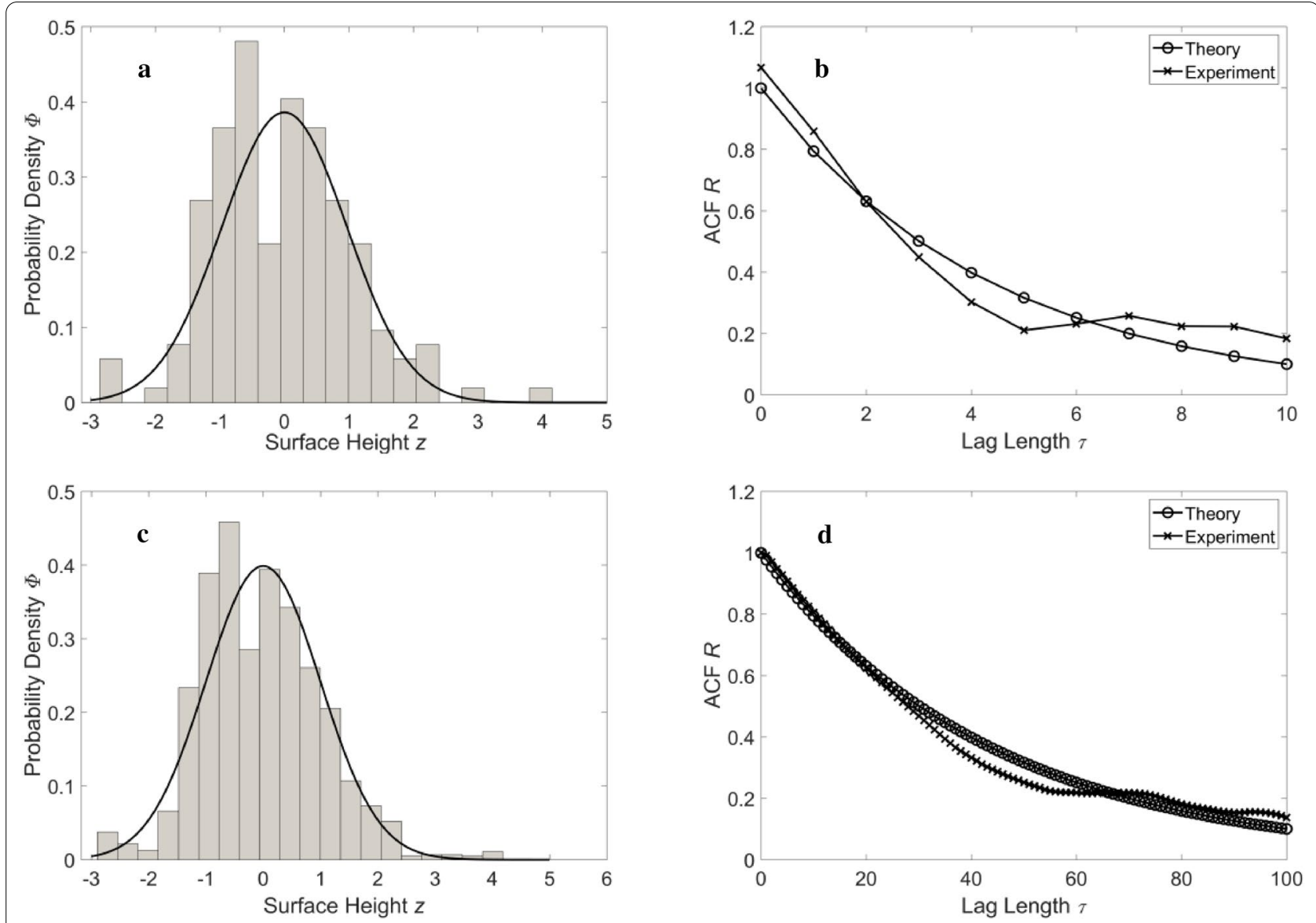

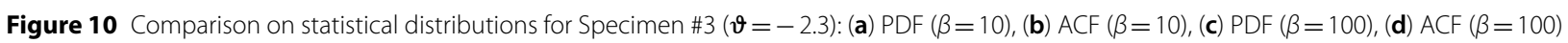
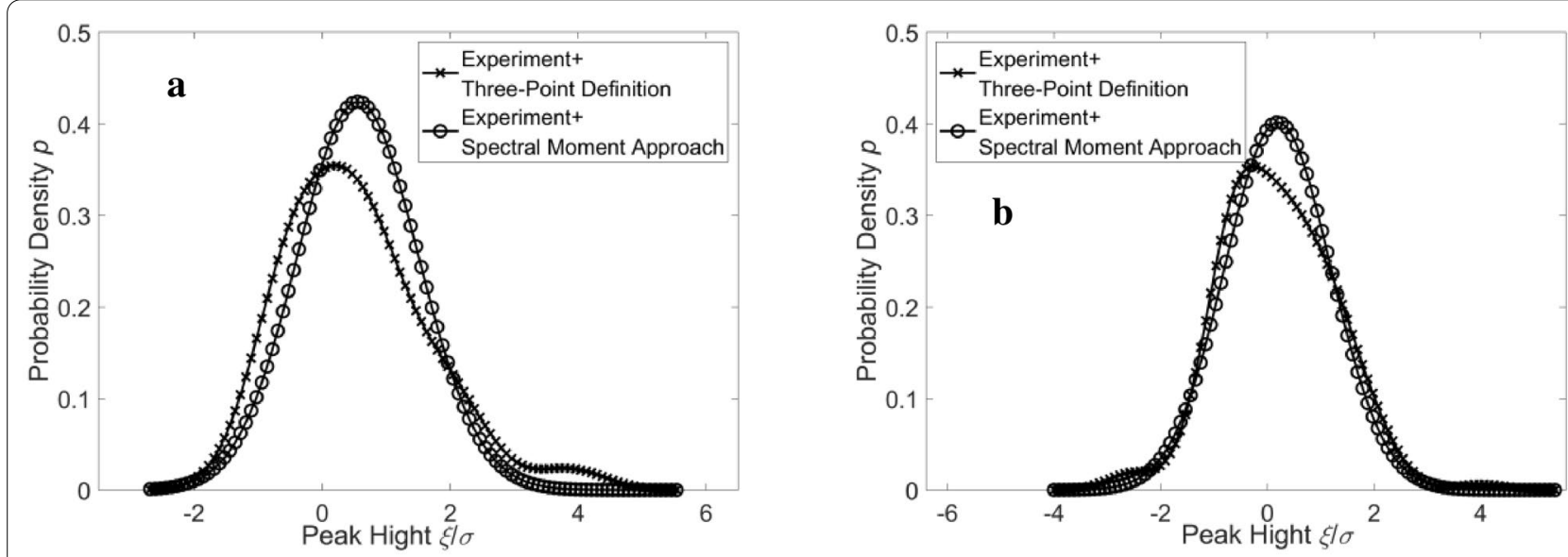

Figure 11 Comparison on PDF of [द] for Specimen \#3: (a) $\beta=10$, (b) $\beta=100$ 

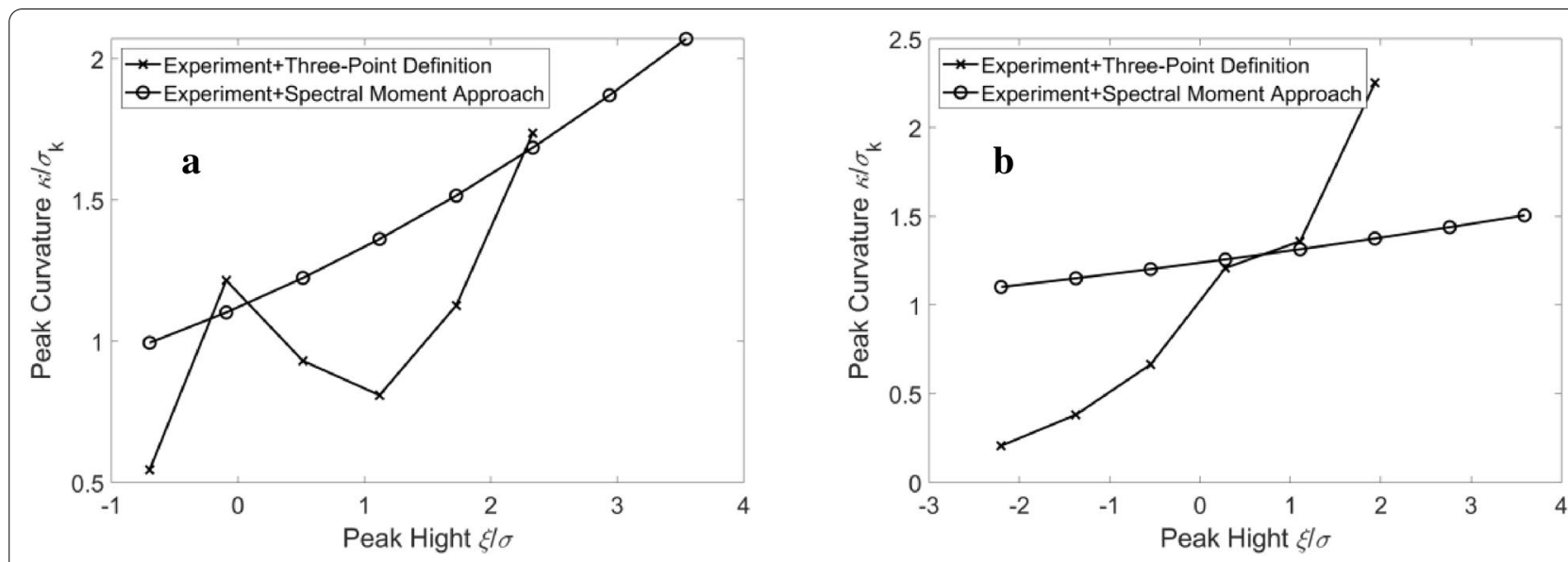

Figure 12 Comparison on curvature distribution of asperity peaks for Specimen \#3: (a) $\beta=10$, (b) $\beta=100$

Table 2 Comparison of $\xi_{\mathrm{avg}}$ and $\bar{\kappa}$ for Specimen \#3 $(\sigma=1, \vartheta=-2.3)$

\begin{tabular}{llllll}
\hline Correlation length & Method & $\boldsymbol{\xi}_{\text {avg }}$ & Percent variation & $\overline{\boldsymbol{\kappa}}$ & Percent variation \\
\hline 10 & Three-point definition & 0.5589 & 0 & 1.2285 & 0 \\
& Spectral moment approach & 1.0816 & $93.52 \% \uparrow$ & 1.3519 & $10.04 \% \uparrow$ \\
\multirow{2}{*}{100} & Three-point definition & 0.1977 & 0 & 1.1205 & 0 \\
& Spectral moment approach & 0.3493 & $76.68 \% \uparrow$ & 1.2617 & $12.60 \% \uparrow$ \\
\hline
\end{tabular}
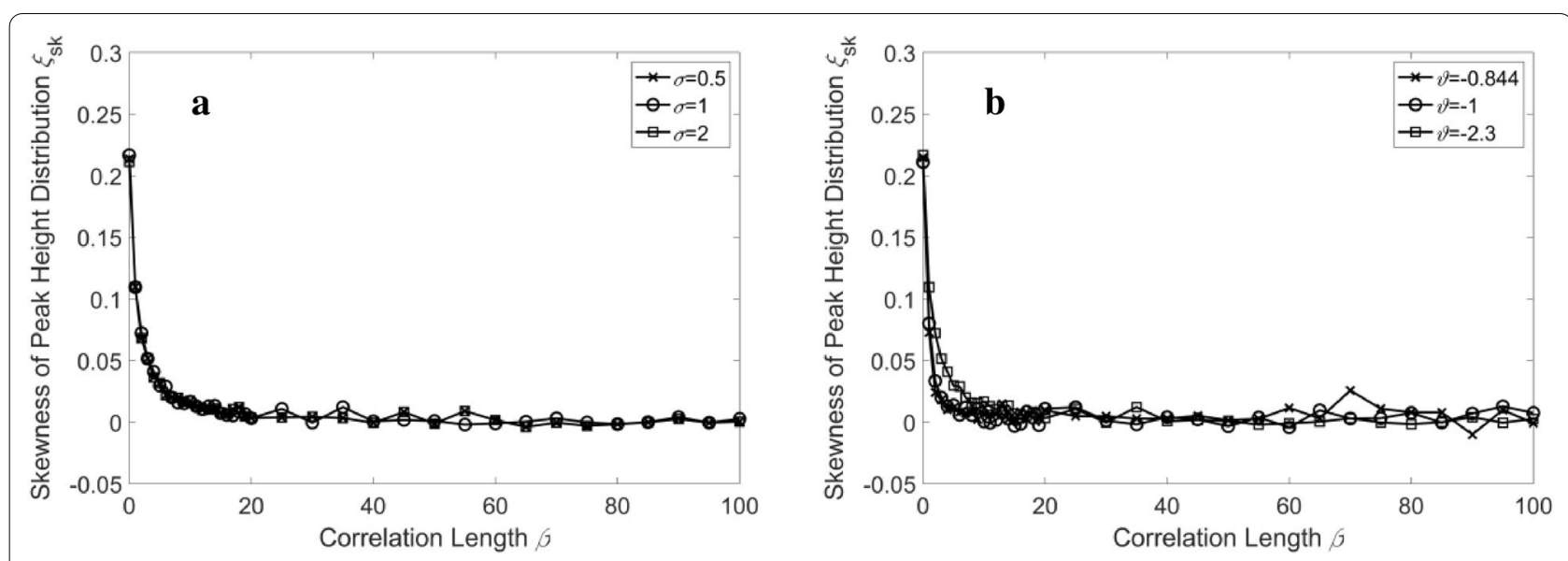

Figure 13 Variations of $\xi_{\text {sk }}$ with correlation length: (a) $\vartheta=-2.3,(\mathbf{b}) \sigma=1$ 

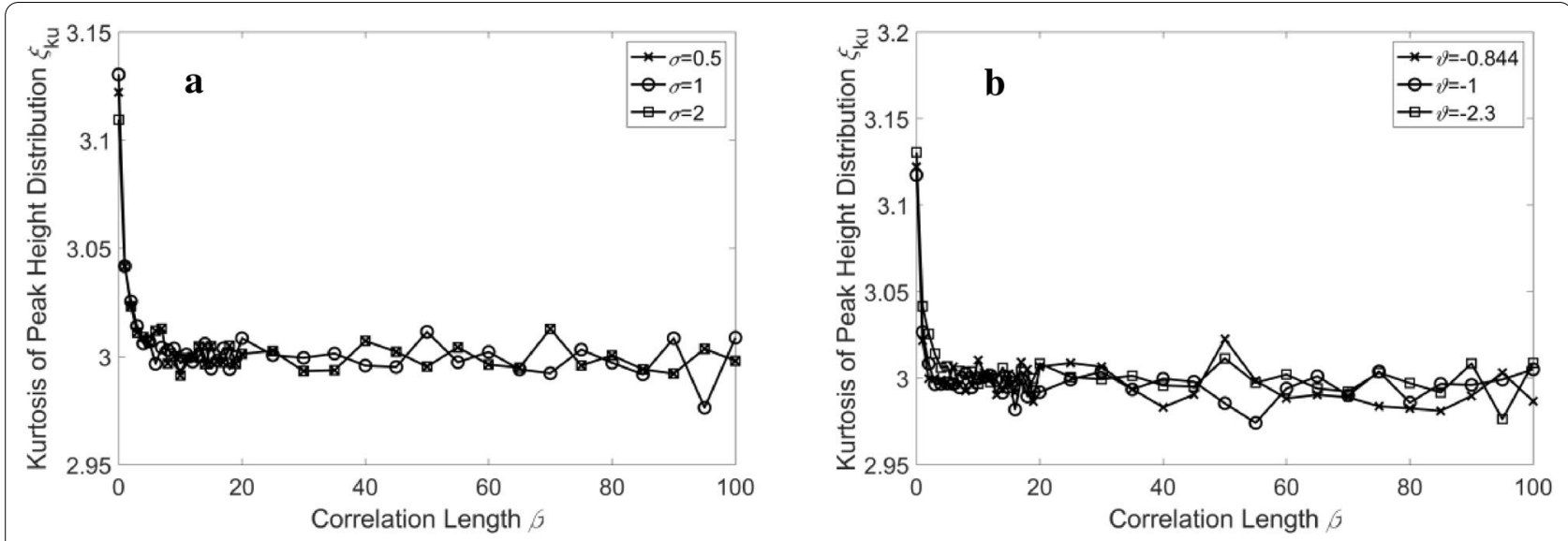

Figure 14 Variations of $\xi_{\text {ku }}$ with correlation length: (a) $\vartheta=-2.3,(\mathbf{b}) \sigma=1$
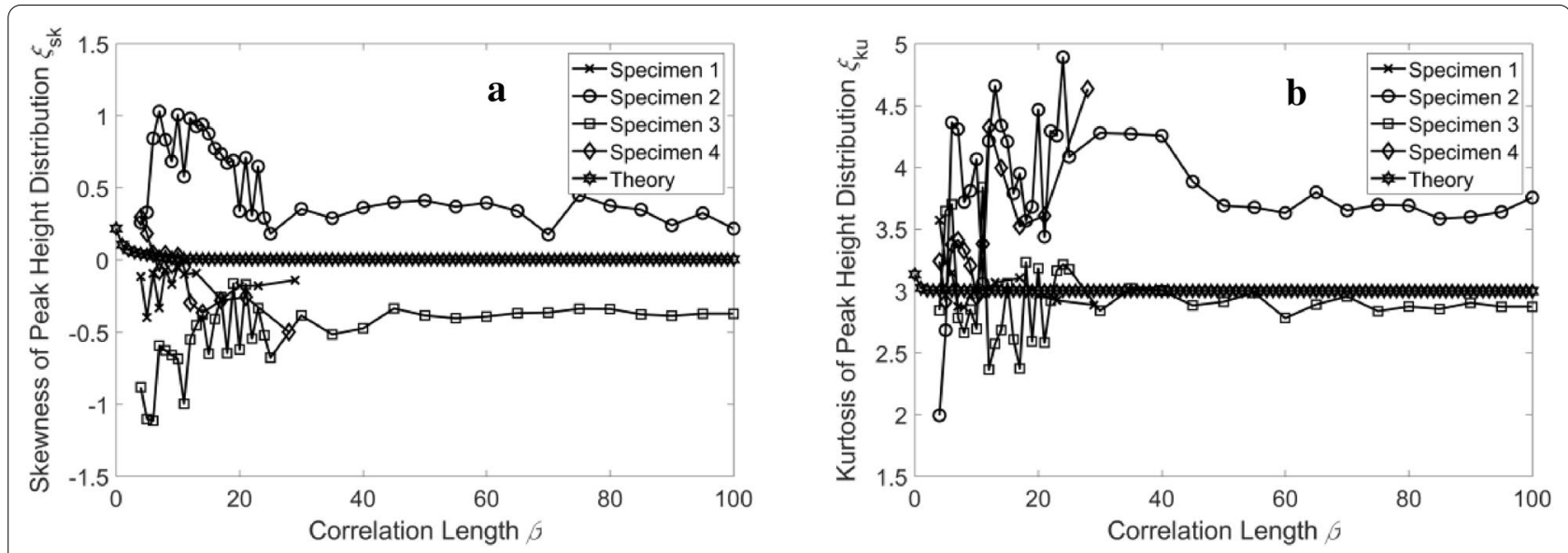

Figure 15 Comparison of peak height distribution parameters between theory and experiment $(\vartheta=-2.3)$ : (a) $\xi_{\text {sk }}$ (b) $\xi_{\text {ku }}$

$$
\phi\left(\xi_{1} \cdots \xi_{n}\right)=(2 \pi)^{-n / 2}\left(\operatorname{Det} .\left(N_{i j}\right)\right)^{-1 / 2} \exp \left\{-\frac{1}{2} M_{i j} \xi_{i} \xi_{j}\right\}
$$

where $\xi_{1}=z, \xi_{2}=\mathrm{d} z / \mathrm{d} x, \xi_{3}=\mathrm{d}^{2} z / \mathrm{d} x^{2}$

$$
N=\left(\begin{array}{cccc}
\overline{\xi_{1}^{2}} & \overline{\xi_{1} \xi_{2}} & \cdots & \overline{\xi_{1} \xi_{n}} \\
\overline{\xi_{2} \xi_{1}} & \overline{\xi_{2}^{2}} & \cdots & \overline{\xi_{2} \xi_{n}} \\
\vdots & \vdots & \ddots & \vdots \\
\overline{\xi_{n} \xi_{1}} & \overline{\xi_{n} \xi_{2}} & \cdots & \overline{\xi_{n}^{2}}
\end{array}\right), \quad \overline{\xi_{i} \xi_{j}}=\int \cdots \iint \xi_{i}\left(\varepsilon_{1}, \cdots, \varepsilon_{k}\right) \xi_{j}\left(\varepsilon_{1}, \cdots, \varepsilon_{k}\right) \phi\left(\varepsilon_{1}\right) \cdots \phi\left(\varepsilon_{k}\right) \mathrm{d} \varepsilon_{1} \cdots \mathrm{d} \varepsilon_{k}, M=(N)^{-1}
$$

research of Sklar [26], any multivariate joint distribution can be written in terms of univariate marginal distribution functions and a copula which describes the dependence structure between quantities. There are $\varepsilon$ is an independent random variable upon which $\xi$ depends.

The question is that even if $[z],\left[z^{\prime}\right]$ and $\left[z^{\prime \prime}\right]$ are all Gaussian, their joint distribution is not necessarily a normal distribution as assumed. According to the many parametric copula families available to obtain the best fitted empirical copula functions [27]. Hence, the mathematic formulae of asperity peak distribution parameters deduced from Eq. (18) may be theoretically inaccurate to a certain extent. 

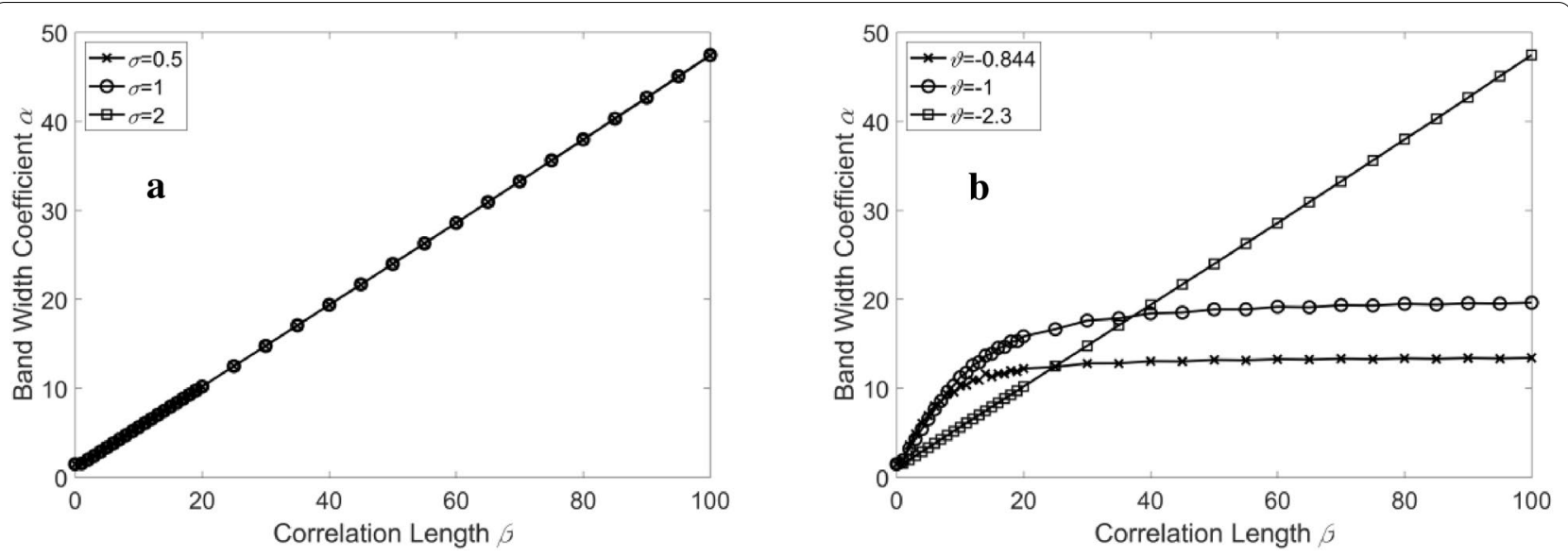

Figure 16 Variations of $a:(\mathbf{a}) \vartheta=-2.3,(\mathbf{b}) \sigma=1$

In the meantime, another assumption that the height distribution of asperities is Gaussian is made to calculate the mean and the RMS of $[\xi]$. In this case, the skewness $\xi_{\mathrm{sk}}$ and the kurtosis $\xi_{\mathrm{ku}}$ of $[\xi]$ should be 0 and 3 respectively. To examine the rationality of this assumption, $\xi_{\mathrm{sk}}$ and $\xi_{\mathrm{ku}}$ of the generated samples are calculated. The variations of the two parameters with correlation length $\beta$ are shown in Figure 13 and Figure 14 respectively. As can be seen, with the increase of $\beta, \xi_{\mathrm{sk}}$ and $\xi_{\mathrm{ku}}$ decrease gradually and finally tend to be 0 and 3 respectively. This indicates that the assumption of $[\xi]$ being Gaussian is exactly correct only with $\beta$ increasing to a certain extent, e.g., greater than 20 . This is consistent with the experiment results shown in Figure 15.

In the work of Nayak [9], band width coefficient $\alpha$ was used to depict the extent to which $[\xi]$ was close to Gaussian distribution. When $\operatorname{Lim} \alpha \rightarrow 1$, there follows

$$
\phi_{\text {peak }}\left(\xi^{*}\right)= \begin{cases}\xi^{*} \exp \left(-1 / 2 \xi^{* 2}\right), & \xi^{*}>0, \\ 0, & \xi^{*}<0 .\end{cases}
$$

It is clear that $[\xi]$ is obviously not Gaussian. When $\operatorname{Lim} \alpha \rightarrow \infty,[\xi]$ becomes

$$
\phi_{\text {peak }}\left(\xi^{*}\right)=\frac{1}{\sqrt{2 \pi}} \exp \left(-1 / 2 \xi^{* 2}\right) .
$$

At this moment, $[\xi]$ follows Gaussian distribution.

Figure 16 shows that $\alpha$ depends on $\beta$ and is irrelevant to $\sigma$. When the effect of high pass filtering is not considered, whether $[\xi]$ is Gaussian is completely determined by $\beta$. Since $\alpha$ increases with $\beta,[\xi]$ is closer to Gaussian distribution under a larger $\beta$. This is in accordance with the modeling difference variation of $[\xi]$ shown in Figure 2 . Thus, increasing $\beta$ by reducing sampling interval $d$

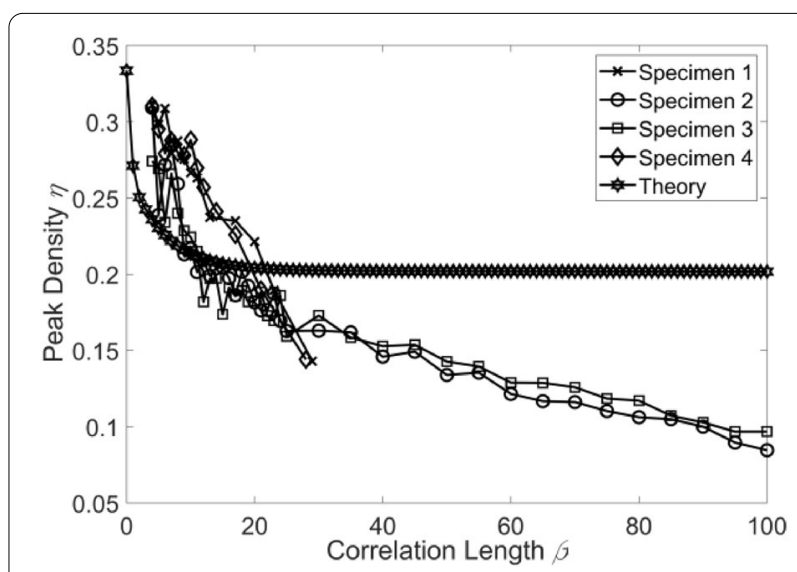

Figure 17 Comparison on asperity peak density between theory and experiment

helps to obtain rough surfaces with $[\xi]$ of Gaussian height distribution. Compared with $\alpha \rightarrow \infty$ by spectral moment approach, $[\xi]$ of satisfactory Gaussian distribution will be achieved for $\beta>20$ according to three-point definition as demonstrated in Figure 13 and Figure 14. Moreover, although Figure 3, Figure 5 and Figure 7 state that high pass filtering has great effect on $[\xi]$, the gaussianity of $[\xi]$ seems not to be influenced by $\vartheta$ as illustrated in Figure 14 and Figure 15.

As pointed out by Whitehouse and Archard [19] that the statistical properties of a surface are not its intrinsic properties but vary with sampling interval. Reducing sampling interval $d$ leads to more points sampled in the same sampling length $L$, which results in the increasement of non-dimensionalized correlation length $\beta$. As a result, the mean $\xi_{\text {avg }}$ and the RMS $\sigma_{\xi}$ of $[\xi]$ decreases and grows respectively. In the meantime, more asperity 
Table 3 Comparison of [द] for surfaces with negative skewness $(\sigma=1, \vartheta=-2.3)$

\begin{tabular}{|c|c|c|c|c|c|c|}
\hline Skewness & Correlation length & Method & $\xi_{\mathrm{avg}}$ & $\sigma$ & $\bar{\kappa}$ & $\eta$ \\
\hline \multirow[t]{6}{*}{-0.25} & 0 & Three-point definition & 0.8281 & 0.9931 & 1.1637 & 0.3330 \\
\hline & & Spectral moment approach & 1.8214 & 0.6360 & 1.2382 & 0.2093 \\
\hline & 50 & Three-point definition & 0.2639 & 1.0830 & 1.2095 & 0.1971 \\
\hline & & Spectral moment approach & 0.5281 & 1.0492 & 1.2715 & 0.1751 \\
\hline & 100 & Three-point definition & 0.2477 & 1.0463 & 1.2027 & 0.1998 \\
\hline & & Spectral moment approach & 0.4235 & 1.1686 & 1.2940 & 0.1749 \\
\hline \multirow[t]{6}{*}{-0.5} & 0 & Three-point definition & 0.8268 & 0.9953 & 1.0846 & 0.3353 \\
\hline & & Spectral moment approach & 1.8412 & 0.6351 & 1.2884 & 0.2094 \\
\hline & 50 & Three-point definition & 0.2151 & 1.0449 & 1.1001 & 0.2041 \\
\hline & & Spectral moment approach & 0.5093 & 1.0311 & 1.2142 & 0.1752 \\
\hline & 100 & Three-point definition & 0.2102 & 1.0928 & 1.1747 & 0.1927 \\
\hline & & Spectral moment approach & 0.3691 & 1.0696 & 1.2243 & 0.1742 \\
\hline
\end{tabular}

peaks are obtained, yet the asperity peak density $\eta$ tends to decrease. The curvature $\kappa$ of $[\xi]$ are decided by the combination effects of increasing $\beta$ and reducing $d$. To diminish the scale dependence, a small enough sampling interval along with a fitting strategy is recommended [6].

Furthermore, since the standard deviation $\sigma$ of $[z]$ varies approximately as $L^{1 / 2}$, rough surfaces are usually treated as stationary stochastic processes, and the influences of sampling length can be ignored.

As a note, the average curvature radius of asperity peaks $r_{\mathrm{p}}$ is calculated with the mean $\xi_{\text {avg }}$ of $[\xi]$ based on Eq. (14), instead of being weighted averaged with $[\xi]$ for spectral moment approach. Nevertheless, such an approximate treatment does not violate the truth that spectral moment approach overestimates the asperity peak curvature as shown in Figure 4, and hence it does not affect the conclusion that the average curvature radius of asperity peaks is underestimated by spectral moment approach.

The comparison of the two methods certainly can be extended to three dimensional surface where asperity summit instead of asperity peak is concerned. In this paper, asperity peak density is found to be underestimated by spectral moment approach. However, Pawar et al. [11] arrived at a conclusion that spectral moment approach overestimated asperity summit density, which was also reported by Kalin et al. [14]. The conflict may result from the fact that not only asperity peaks but also asperity shoulders were considered when asperity summits were modeled. The ACFs of the specimens prepared by Kalin could deviate from ACFs in theory as depicted in Figure 10. For this reason, the calculated asperity peak/summit density of specimens can be different from those of theory, as shown in Figure 17.
As surfaces with negative skewness are common, it is meaningful to find out whether the aforementioned differences between the two methods change for such surfaces. Table 3 gives the comparison results using simulated rough surface profile samples of negative skewness. The result shows that the differences between the two methods almost have similar variations whatever skewness is.

Finally, it should be emphasized that geometry modeling of asperity is focused in this paper. The coalescing of asperities during contact is not considered [28, 29]. In this sense, the peak identification scheme based method, i.e., threepoint definition, and a small enough sampling interval along with a fitting strategy should be adopted to accurately model asperity peak distribution for tribological problems.

\section{Conclusions}

(1) For rough surfaces of Gaussian height distribution and exponential ACF common in engineering, spectral moment approach overestimates the average height and the average curvature of asperity peaks and underestimates the RMS of asperity peak height distribution, the average curvature radius of asperity peaks and asperity peak density, as compared with three-point definition.

(2) The asperity modeling differences between spectral moment approach and three-point definition are validated by experiment and are found to mainly depend upon standard deviation and correlation length. High pass filtering constant has little influence on the differences between the two methods.

(3) The fact that the joint distribution of surface height, slope and curvature is probably not normal according to their correlation pattern and that the height distribution of asperities is not exactly Gaussian 
at small correlation lengths, may account for the discovered differences between spectral moment approach and three-point definition.

\section{Acknowledgements \\ Not applicable.}

\section{Authors' Contributions}

WZ was in charge of the whole study; WZ and DZ wrote the manuscript: JT and JY assisted with analysis. All authors read and approved the final manuscript.

\section{Authors' Information}

Wei Zhou, born in 1985, is currently an assistant professor at Hunan University of Science and Technology, China. He received his PhD degree from Central South University, China, in 2016. His research interests include structural fatigue and tribology.

Daiyan Zhao, born in 1997, is currently a master candidate at Hunan University of Science and Technology, China.

Jinyuan Tang, born in 1962, is currently a professor and a PhD candidate supervisor at Central South University, China. His research interests include digital design and manufacture.

Jun Yi, born in 1987, is currently an assistant professor at Hunan University of Science and Technology, China. He received his PhD degree from Hunan University, China, in 2017. His research interests include high efficiency and precision machining of difficult-to-cut materials and complex curved parts.

\section{Funding}

Supported by National Natural Science Foundation of China (Grant Nos. 51705142, 51535012) and Hunan Provincial Natural Science Foundation of China (Grant No. 2018JJ3162).

\section{Competing interests}

The authors declare no competing financial interests.

\section{Author Details}

${ }^{1}$ Hunan Provincial Key Laboratory of High Efficiency and Precision Machining of Difficult-to-Cut Material, Hunan University of Science and Technology, Xiangtan 411201, China. ${ }^{2}$ State Key Laboratory of High Performance Complex Manufacturing, Central South University, Changsha 410083, China.

Received: 12 May 2020 Revised: 24 January 2021 Accepted: 28 May 2021 Published online: 09 June 2021

\section{References}

[1] V L Popov, R Pohrt. Friction and Wear: From Elementary Mechanisms to Macroscopic Behavior. Lausanne: Frontiers Media, 2019.

[2] Y Q Wen, JYTang, W Zhou. Influence of distribution parameters of rough surface asperities on the contact fatigue life of gears. Proceedings of the Institution of Mechanical Engineers, Part J: Journal of Engineering Tribology, 2019, 234(6): 821-832.

[3] K Komvopoulos. A multiscale theoretical analysis of the mechanical, thermal, and electrical characteristics of rough contact interfaces demonstrating fractal behavior. Frontiers in Mechanical Engineering, 2020, 6: 36.

[4] J A Greenwood, J B P Williamson. Contact of nominally flat surfaces. Proceedings of the Royal Society of London Series A Mathematical and Physical Sciences, 1966, 295: 300-319.

[5] Y Q Wen, JYTang, W Zhou, et al. A reconstruction and contact analysis method of 3-D rough surface based on ellipsoidal asperity. Journal of Tribology, 2020, 142: 041502.
[6] Y Q Wen, JYTang, W Zhou, et al. An improved simplified model of rough surface profile. Tribology International, 2018, 125: 75-84.

[7] H Deng, Z Xu, L Q Wang, et al. Laser micro-structuring of a coarse-grained diamond grinding wheel. International Journal of Advanced Manufacturing Technology, 2019, 101: 2947-2954.

[8] M S Longuet-Higgins. Statistical properties of an isotropic random surface. Philosophical Transactions of the Royal Society A Mathematical Physical \& Engineering Sciences, 1957, 250: 157-174.

[9] P R Nayak. Random process model of rough surfaces. Journal of Lubrication Technology, 1971, 93: 398-407.

[10] J I McCool. Comparison of models for the contact of rough surfaces. Wear, 1986, 107: 37-60.

[11] G Pawar, P Pawlus, I Etsion. The effect of determining topography parameters on analyzing elastic contact between isotropic rough surfaces. Journal of Tribology, 2013, 135: 011401

[12] W Zhou, JYTang, Y F He. Formulae of roughness peak distribution parameters with standard deviation and correlation length. Proceedings of the Institution of Mechanical Engineers, Part J Journal of Engineering Tribology, 2015, 229: 1395-1408.

[13] A Pogačnik, M Kalin. How to determine the number of asperity peaks, their radii and their heights for engineering surfaces: A critical appraisal. Wear, 2013, 300: 143-154.

[14] M Kalin, A Pogačnik, I Etsion. Comparing surface topography parameters of rough surfaces obtained with spectral moments and deterministic methods. Tribology International, 2016, 93: 137-141.

[15] S Panda, A Panzade, M Sarangi, et al. Spectral approach on multiscale roughness characterization of nominally rough surfaces. Journal of Tribology, 2016, 139: 031402.

[16] W R Chang, I Etsion, D B Bogy. An elastic-plastic model for the contact of rough surfaces. Journal of Tribology, 1987, 109: 257-263.

[17] S Hu, W Huang, N Brunetiere, et al. Stratified effect of continuous biGaussian rough surface on lubrication and asperity contact. Tribology International, 2016, 104: 328-341.

[18] X Zhang, Y Xu, R L Jackson. An analysis of generated fractal and measured rough surfaces in regards to their multi-scale structure and fractal dimension. Tribology International, 2017, 105: 94-101.

[19] D J Whitehouse, J F Archard. The properties of random surfaces of significance in their contact. Proceedings of the Royal Society of London Series A Mathematical and Physical Sciences, 1970, 16: 97-121.

[20] W Hirst, A E Hollander. Surface finish and damage in sliding. Proceedings of the Royal Society of London Series A Mathematical and Physical Sciences, 1974, 337: 379-394.

[21] H Aramaki, H S Cheng, Y W Chung. The contact between rough surfaces with longitudinal texture. I: Average contact pressure and real contact area. Journal of Tribology, 1993, 115: 419-424.

[22] A Francisco, N Brunetiere. A hybrid method for fast and efficient rough surface generation. Proceedings of the Institution of Mechanical Engineers, Part J Journal of Engineering Tribology, 2016, 230(7): 747-768.

[23] Y F He, JYTang, W Zhou. Research on the obtainment of topography parameters by rough surface simulation with fast Fourier transform. Journal of Tribology, 2015, 137: 031401.

[24] B Sista, KVemaganti. Estimation of statistical parameters of rough surfaces suitable for developing micro-asperity friction models. Wear, 2014 316: 6-18.

[25] TR Thomas. Rough surfaces. London: Imperial Coll Press, 1999.

[26] A Sklar. Random variables, joint distribution functions, and copulas. Kybernetika Praha, 1973, 9: 449-460.

[27] U Cherubini, B Luciano, W Vecchiato. Copula methods in finance. England: John Wiley \& Son, 2004.

[28] P R Nayak. Random process model of rough surfaces in plastic contact. Wear, 1973, 26: 305-333.

[29] J A Greenwood. A note on Nayak's third paper. Wear, 2007, 262: 225-227. 